\title{
ANÁLISE DE RISCO DO DESEMPENHO ECONÔMICO DE UM SISTEMA DE RECRIA DE GADO DE CORTE EM REGIME DE PASTEJO ROTACIONADO ${ }^{1}$
}

\author{
Andre Rozemberg Peixoto Simões ${ }^{2}$ \\ Altair Dias de Moura
}

Resumo - Os resultados técnicos e econômicos dos sistemas de produção agropecuários estão condicionados por uma série de variáveis sociais, culturais e macroeconômicas que limitam ou impulsionam o dinamismo do setor. Com vistas em obter rentabilidade, os produtores devem procurar ganhos de produtividade e aumento de produção. Desta forma, o processo de profissionalização das atividades agropecuárias passa pela adoção de técnicas modernas de gerenciamento e de tomadas de decisão. Este trabalho objetivou exercitar técnicas de análise econômica, análise de sensibilidade, geração de cenários e de análise de risco. Foram utilizados dados primários de um sistema de recria de gado de corte em regime de pastejo rotacionado na aplicação dos métodos. Os resultados revelaram que o sistema estudado é lucrativo, entretanto, as análises de cenários e de risco indicam baixa atratividade e elevada probabilidade de obtenção de lucros negativos.

Palavras-chave: Análise econômica, gado de corte, cenários, simulação de Monte Carlo, análise de risco.

\section{Introdução}

A década de 90 e o início do ano 2000 foram marcados por um importante processo de mudanças na economia brasileira. Na esfera comercial, verificou-se a abertura do país ao mercado exterior, calcado na queda de tarifas e na desregulamentação do mercado. O maior fluxo comercial de bens, advindo da abertura comercial, foi responsável pela nova ordem de competição do agronegócio.

\footnotetext{
Recebido em 03/10/2005 Aceito em 25/01/2006.

Professor da Universidade Estadual de Mato Grosso do Sul - UEMS. E-mail: andrerpsimoes@uems.br

3 Professor do Departamento de Economia Rural da Universidade Federal de Viçosa. E-mail: admoura@ufv.br
} 
A partir desse período, os produtores rurais, indústrias de processamento e rede de varejistas tiveram que se reestruturar, para produzir, comercializar e manter uma coordenação vertical mais eficiente. Somente com essa nova estrutura que se desenhou no cenário do agronegócio é que o Brasil pôde competir, tanto interna como externamente, com os produtos de outros países (Simões, 2003).

O país tem conseguido atingir elevados índices de competitividade no mercado internacional de carne bovina (Reis, 2003); entretanto, as exigências dos países compradores têm se intensificado. As barreiras tarifárias presentes no comércio internacional têm sido o cerne da discussão das rodadas de negociações internacionais da OMC. Dessa forma, surgem novas tendências de proteção à entrada de produtos importados na forma de exigência de consumidores e normas de segurança do alimento.

Crises sanitárias internacionais, como febre aftosa, contaminações por dioxina e mal da vaca louca, aliadas à crescente preocupação com a preservação do meio ambiente, têm gerado demanda de alimentos produzidos por processos naturais e não agressivos ao meio ambiente. A saúde do homem nunca esteve tanto em evidência, devido à ingestão de produtos não seguros e pouco saudáveis.

Nas últimas décadas, têm surgido novos conceitos de sustentabilidade dos sistemas produtivos, os quais se fundamentam no enfoque social, econômico e, mais recentemente, no enfoque ambiental.

Tal delineamento desta nova demanda tem gerado a necessidade de mudança nos processos produtivos, nas instâncias dos primeiros elos da cadeia produtiva, ou seja, o produtor rural. Tal demanda exige que os animais sejam criados naturalmente, na ausência de pesticidas, antibióticos, hormônios ou quaisquer outros resíduos nocivos ao meio ambiente e ao próprio homem que os consome. 
O respeito pelos ecossistemas e a preservação do meio ambiente representam pontos de grande importância na construção da imagem do produto no exterior. As novas versões do "Boi Verde" e do "Boi Orgânico 5" são temas já bem conhecidos e muito debatidos na Europa e em outros mercados internacionais. Embora os pecuaristas brasileiros tenham que continuar aumentando a produtividade para permanecerem rentáveis, agora eles devem fazê-lo de maneira ambientalmente correta (Bonjour, 2003).

O Brasil possui vantagens comparativas e grande potencial produtivo a ser explorado, em relação aos animais criados sob o regime de pastoreio; entretanto, novas formas sustentáveis de manejo dos recursos naturais devem ser estudadas, com o intuito de preservar e melhorar as características do meio ambiente.

Diante desse ambiente composto por inúmeras variáveis que afetam o desempenho da pecuária nacional, a classe produtora, freqüentemente, questiona os baixos preços pagos e os elevados custos dos insumos, o que leva à redução na lucratividade. Tais variações conjunturais dos termos de troca podem ser minimizadas por várias ações, dentre elas, a integração horizontal dos pecuaristas para negociação com os setores a montante e a jusante. Esta ação pode trazer melhorias no ambiente empresarial, porém os efeitos das variáveis exógenas, como taxa de câmbio, cotações internacionais da carne bovina, preço das carnes concorrentes, mudanças no ambiente macroeconômico e político e exigências dos consumidores, afetam a empresa rural de modo sistemático.

Dessa maneira, torna-se necessário que o empresário rural utilize técnicas de apoio à decisão que minimizem os riscos de sua atividade e levem a resultados econômicos satisfatórios.

\footnotetext{
Boi alimentado em pastagem com ou sem adubação química, permitindo-se o uso de antibióticos e de outros medicamentos alopáticos. A suplementação alimentar só pode ser feita com produtos de origem vegetal. Era chamado de boi extensivo.

5 Boi alimentado em pastagem sem adubação química e tratado exclusivamente com medicamentos homeopáticos, sendo proibido o uso de antibióticos. A suplementação alimentar só pode ser feita com produtos orgânicos.
} 
Os efeitos das alterações do ambiente institucional levam a modificações estruturais na competitividade das fazendas produtoras de carne, razão pela qual devem ser pensadas ações que alterem a tecnologia atual para obter ganhos de produtividade e melhoria na alocação dos recursos.

O pastejo rotacionado de animais é um sistema de produção que preconiza a intensificação da utilização do recurso terra por meio do aumento na pressão de pastejo, o que gera maior ganho de carne produzida por unidade de área.

Neste sistema, o consumo de suplementos concentrados é relativamente baixo, sendo a maior parte das exigências nutricionais dos animais satisfeita pelo próprio pasto. $\mathrm{O}$ aumento no uso de fertilizantes possibilita uma carga animal superior ao pastejo contínuo e ainda protege o fator terra e as pastagens da degradação.

O sistema de pastejo rotacionado atende às exigências dos consumidores mais exigentes, tanto na qualidade e na segurança do alimento quanto na preservação ambiental, uma vez que possibilita incrementos de produção em áreas já estabelecidas e não preconiza a abertura de novas áreas de mata nativa. Finalmente, o pastejo rotacionado apresenta-se como alternativa para maiores ganhos de eficiência técnica e econômica da atividade pecuária.

A pecuária de corte no Estado de Mato Grosso do Sul tem passado por uma transição de pouca tecnificação e de gestão administrativa ineficiente para uma atividade empresarial e competitiva. A gestão das informações e a utilização de técnicas modernas de tomadas de decisão são cada vez mais freqüentes neste ramo.

O ambiente de incertezas, a volatilidade dos preços de insumos e produtos e a falta de informações precisas dificultam a tomada de decisão e inibe novos investimentos no setor produtivo de gado de corte.

Assim, a metodologia de análise econômica de sistemas de produção, associada à gestão dos riscos, pode auxiliar na tomada de decisão dos 
pecuaristas. Este trabalho objetivou exercitar e difundir técnicas de análise econômica, análise de sensibilidade, geração de cenários e de análise de risco para sistemas de produção de gado de corte.

Especificamente, objetivou-se utilizar tais técnicas para analisar um sistema de recria de gado de corte em regime de pastejo rotacionado.

\section{Antecedentes}

Na literatura, pode-se identificar uma série de trabalhos que se propuseram a fazer análise de viabilidade econômica associada à de risco. Dentre os consultados, destacam-se os de Souza (2001), analisou o risco econômico aplicado a sistemas de irrigação para cultura cafeeira.; Ponciano et al. (2003), que fizeram uma análise de viabilidade econômica e de risco para projetos de fruticultura no Norte do Estado do Rio de Janeiro; e Almeida et al. (2003), que avaliaram a viabilidade de implantação de um projeto de produção de polpa de fruta em Campo dos Goytacazes - RJ.

Mais especificamente em trabalhos com pastagens na mesma linha de pesquisa, destacam-se os de Maya (2003), que desenvolveu uma análise comparativa de viabilidade técnica, econômica e de risco entre manejos irrigado e não-irrigado, e de Scolari (1988), que fez uma análise econômica da produção de carne bovina na região do Cerrado, testando diferentes tipos de pastagens.

\section{Metodologia}

Para cumprir os objetivos propostos foram calculados indicadores de viabilidade econômica e, em seguida, foram gerados possíveis cenários para estes indicadores, a partir do coeficiente de variação de algumas variáveis. Na última etapa, utilizou-se o método de Monte Carlo na simulação de risco, a partir das mesmas variáveis utilizadas na geração dos cenários. Um resumo das etapas pode ser visualizado na Figura 1. 


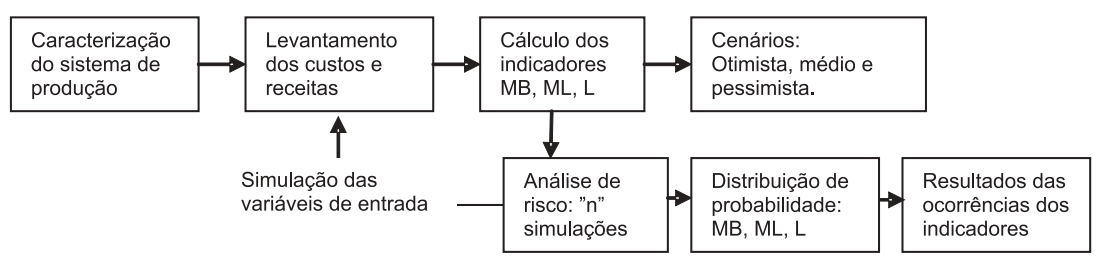

Figura 1 - Resumo das etapas seguidas no trabalho.

Fonte: Elaborado pelos autores.

\subsection{Fonte de dados}

Este trabalho utilizou dados primários de um sistema de produção de recria de machos implantado na Fazenda CERA/UEMS, em Aquidauana - MS, onde funciona a unidade da Universidade Estadual de Mato Grosso do Sul, unidade Aquidauana. Foi utilizada uma área de pastagem com cerca de 10 hectares, onde foram estabelecidos dois blocos com doze piquetes de 0,4 cada. Os piquetes foram formados de braquiaria decumbens, com utilização de calcário para correção da acidez, e uma adubação orgânica elaborada na própria fazenda. Foram utilizados 40 machos nelores, que entraram no sistema com peso médio de $180 \mathrm{Kg}$ e saíram com peso médio de $285 \mathrm{Kg}$. Os piquetes foram cercados com cerca elétrica. A alimentação foi basicamente de pasto e sal mineral. Os preços foram coletados na praça de Aquidauana - MS e corrigidos pelo IGP-DI (Ipeadata, 2005), para o mês de fevereiro de 2005.

Alguns valores dos custos de produção, como o gasto com mão-de-obra contratada, e de algumas benfeitorias foram estimados, pois a estrutura da fazenda da UEMS não representa as necessidades do sistema de produção trabalhado. Não foram computadas depreciações para maquinário, pois estas foram contratadas por hora trabalhada no mercado local.

O modelo de análise econômica elaborado apontou três variáveis principais que afetavam os resultados, quais sejam, o preço de compra dos bezerros, o preço de venda dos animais no final do ciclo e os gastos 
com mão-de-obra. Tais variáveis foram consideradas, o que significa que os cenários e a análise de risco foram desenvolvidos pela variação nos valores destas.

Os dados referentes às variáveis de entrada foram obtidos da Federação da Agricultura do Mato Grosso do Sul - FAMASUL, e do IPEA. Foram coletados preços diários de compra dos bezerros no período de junho de 2001 a fevereiro de 2005, e os preços de venda dos garrotes referiramse ao período de fevereiro 2003 a fevereiro 2005. Os dados referentes a despesas com mão-de-obra foram indexados pelo salário mínimo e a série utilizada, do período de 1999 a 2004 (dados mensais corrigidos para setembro 2004). A fonte destes dados foi obtida do IPEA e utilizou-se o Microsoft Excel em todos os cálculos.

\subsection{Indicadores econômicos utilizados no modelo}

A classificação básica dos custos de produção, proposta pela teoria microeconômica, é em custos fixos e custos variáveis. Os fixos relacionam-se com fatores de produção, que não podem ser modificados, em termos de quantidade utilizada, em curto período de tempo ou ciclo produtivo. Os fixos independem da quantidade produzida, ou seja, estão presentes na atividade, mesmo que a produção seja zero, e são depreciações, custo de oportunidade do capital, impostos, etc (Varian, 2000).

Os variáveis são associados à utilização de insumos cujas quantidades podem variar em curto espaço de tempo ou em um mesmo ciclo produtivo. Estão relacionados com a quantidade produzida, ou seja, quanto mais se produz, maior é o custo variável; em outras palavras, se nada for produzido, não existirão custos variáveis. São exemplos de custos variáveis as despesas com ração, medicamentos, mão-de-obra, fertilizantes, etc (Varian, 2000). 
Neste trabalho, a classificação dos custos será feita conforme a proposta do Instituto de Economia Agrícola (IEA) de São Paulo, citada por Matsunaga, 1976. Tal classificação permite melhor caracterização do perfil econômico da atividade, bem como possibilita tomada de decisões mais acertadas, em comparação ao método de classificação tradicional.

De acordo com a classificação do IEA, têm-se os seguintes indicadores de desempenho econômico:

$\checkmark$ Receita Total (RT): Comporão a receita todas as entradas monetárias provenientes da venda de animais nos diferentes sistemas de produção.

$\checkmark$ Custo Operacional Efetivo (COE): corresponde aos desembolsos diretos para compra de insumos (concentrados, sal, medicamentos, energia, mão-de-obra contratada, etc.). Compõe os custos variáveis ou do curto prazo.

$\checkmark$ Custo Operacional Total (COT): somada ao COE está a depreciação de máquinas, benfeitorias, culturas não-anuais (pastagens) e mãode-obra familiar, se possuir.

$\checkmark$ Custo total (CT): é a soma do COT mais remuneração do capital médio empatado (custo de oportunidade do capital).

$\checkmark$ Margem Bruta (MB): RT - COE.

$\checkmark$ Margem Líquida (ML): RT - COT.

Lucro (L): RT - CT. 


\subsection{Geração de possíveis cenários}

Após terem sido calculados os indicadores econômicos, foram escolhidas as variáveis que tiveram maior impacto nos resultados. Foram coletadas séries históricas destas variáveis e calculados os valores máximos, mínimos, médios, desvio-padrão e, finalmente, coeficiente de variação $(\mathrm{CV}){ }^{6}$

Os cenários possíveis foram obtidos pelo percentual do CV sobre os valores encontrados nas variáveis de entrada da análise determinística.

\subsection{Análise de risco}

Basicamente, o risco é derivado da incapacidade de prever os eventos futuros, o que torna a decisão do presente um ato de reflexão, ponderação e avaliação das possíveis consequiências futuras dessa decisão (Santos e Vieira, 2003). Dessa forma, conhecer os valores determinísticos dos indicadores econômicos não é suficiente para os tomadores de decisão, pois sua probabilidade de ocorrência pode vir a ser baixa, em períodos futuros.

O primeiro passo para se fazer uma análise de risco é eleger as variáveis que se mostram mais impactantes nos indicadores a serem analisados. Usualmente, faz-se uso da técnica de análise de sensibilidade, que consiste em medir em que magnitude uma alteração prefixada em um ou mais fatores do projeto altera o resultado final. Esse procedimento permite avaliar de que forma as alterações de cada uma das variáveis do projeto podem influenciar na rentabilidade dos resultados esperados (Buarque, 1991).

\footnotetext{
6 Coeficiente de variação expresso em \%.CV=Desvio-padrão / média.
} 
Para avaliar o risco envolvido nos diversos sistemas, será empregada a técnica da simulação de Monte Carlo, que tem sido considerada como ferramenta de grande utilidade pelos tomadores de decisão, ao tratarem de situações sujeitas a risco em seus projetos de investimento. Como destacado por Noronha (1987), esta simulação é, dentre os métodos que utilizam probabilidade na análise dos riscos, o mais simples do ponto de vista prático, além de apresentar custo razoavelmente baixo.

A sequiência de cálculos para realização da simulação de Monte Carlo é a seguinte:

1. Identificar a distribuição de probabilidade de cada uma das variáveis independentes (variáveis de entrada), relevantes na análise.

2. Selecionar, ao acaso, um valor de cada variável, a partir de sua distribuição de probabilidade.

3. Calcular o valor do indicador de escolha, cada vez que for feito o sorteio indicado no item 2 .

4. Repetir o processo até que se obtenha confirmação adequada da distribuição de freqüência do indicador de escolha, o que servirá de base para a tomada de decisão

Os indicadores (ou variáveis de saída) a serem considerados na análise de risco deste trabalho serão Margem Bruta, Margem Líquida e Lucro.

\section{Resultados e discussão}

A discussão dos resultados será dividida em três partes: a primeira diz respeito à análise determinística dos indicadores econômicos encontrados no sistema de produção; a segunda, à análise estática dos cenários gerados; e a terceira, à análise de risco obtida pelo método de Monte Carlo. 


\subsection{Análise determinística dos indicadores econômicos}

Os resultados dos indicadores econômicos, encontrados para o sistema de recria de gado de corte na região de Aquidauana - MS, podem ser vistos, de forma resumida, na Tabela 1 (a descrição detalhada dos custos pode ser vista no apêndice, Tabela 1a).

Tabela 1 - Resumo de renda, custos e indicadores de resultado econômico encontrados para o sistema de produção de recria de machos em pastejo rotacionado na região de Aquidauana - MS, fevereiro 2005

\begin{tabular}{|c|c|c|c|c|}
\hline \multirow[t]{2}{*}{ Especificação } & \multirow[t]{2}{*}{ Unidade } & \multirow{2}{*}{$\begin{array}{l}\text { Total da } \\
\text { Atividade }\end{array}$} & \multicolumn{2}{|c|}{ Total por unidade } \\
\hline & & & $\mathbf{R} \$ / \mathbf{c a b}$ & $\mathbf{R} \$ / \mathbf{h a}$ \\
\hline 1. Receita Total - RB & $\mathrm{R} \$$ & $22.928,00$ & 573,20 & $2.292,80$ \\
\hline \multicolumn{5}{|l|}{ 2. Custo de Produção } \\
\hline 2.1. Custo Operacional Efetivo - COE & $\mathrm{R} \$$ & $20.448,00$ & 511,20 & $2.044,80$ \\
\hline 2.2. Custo Operacional Total - COT & $\mathrm{R} \$$ & $21.897,54$ & 547,44 & $2.189,75$ \\
\hline 2.3. Custo Total - CT & $\mathrm{R} \$$ & $22.652,34$ & 566,31 & $2.265,23$ \\
\hline \multicolumn{5}{|l|}{ 3. Indicadores de Resultado: } \\
\hline 3.1. Margem Bruta (RB-COE) & $\mathrm{R} \$$ & $2.480,00$ & 62,00 & 248,00 \\
\hline 3.2. Margem Líquida (RB-COT) & $\mathrm{R} \$$ & $1.030,46$ & 25,76 & 103,05 \\
\hline 3.3. Lucro (RB-CT) & $\mathrm{R} \$$ & 275,66 & 6,89 & 27,57 \\
\hline
\end{tabular}

Fonte: Dados da pesquisa.

Pode-se notar que a atividade pagou todos seus custos operacionais e apresentou uma Margem Bruta (MB) positiva da ordem de $\mathrm{R} \$ 62,00$ por cabeça, o que sugere continuidade da atividade no curto prazo.

A atividade também se apresentou sustentável no longo prazo, uma vez que os custos com depreciações foram cobertos ${ }^{7}$. A Margem Líquida (ML) positiva indicou que houve formação de capital suficiente para repor os bens de produção no final de suas respectivas vidas úteis.

\footnotetext{
Neste caso, não se incluiu na Margem Líquida a remuneração da mão-de-obra familiar, uma vez que se considerou que toda mão-de-obra necessária havia sido contratada.
} 
O indicador de Lucro (L) positivo indicou que o sistema de produção já era boa alternativa de investimento, pois a receita gerada cobria todos os custos e ainda remunerava o capital médio empatado, a uma taxa superior a considerada de mercado, $6 \%$ ao ano (lucro supernormal).

Especificamente para os indicadores de ML e L, podem-se esperar maiores valores em empreendimentos que possuam maior escala de produção. Há evidências de que podem ocorrer ganhos de escala na utilização da estrutura física e de utilização de mão-de-obra. Assim, espera-se que estes indicadores se tornem mais expressivos.

A análise feita mostra o resultado determinístico, ou seja, sem aleatoriedade de nenhuma variável. Tal fato torna a análise incompleta e com poucos subsídios à tomada de decisão, de forma mais consciente e precisa. Em outras palavras, a análise de indicadores econômicos reflete apenas uma relação técnica entre fatores de produção e o produto gerado, dados os níveis de preços. São excluídos desta análise fatores interferentes nos resultados, como sazonalidade de preços (pagos e recebidos pelo produtor), inflação, demanda de mercado, poder de barganha dos produtores, crises sanitárias, etc.

\subsection{Análise estática dos cenários gerados}

Para superar a limitação da análise anterior, foram propostos alguns cenários possíveis de ocorrer, variando-se algumas variáveis importantes para o modelo. Assim, os cenários foram gerados a partir de diferentes valores das variáveis de entrada: valor unitário de venda dos animais no final do ciclo de recria (receita), valor unitário da compra de bezerros (custo de matéria-prima), e despesas relacionadas com mão-de-obra indexadas pelo salário mínimo (m.d.o. contratada, assistência técnica e contabilidade), por serem estas as que mais impactam os indicadores de saída MB, ML e L. A participação percentual destes itens eleitos (soma totalizando $77,83 \%$ ), em relação à receita total, pode ser vista na Tabela 1a (apêndice). 
Foram gerados 4 cenários para cada indicador econômico do sistema de produção, Gráfico 1.

No cenário 1 (Médio), considerou-se a média dos preços pagos pelos bezerros, dos preços recebidos pela venda dos mesmos animais no final do ciclo e do salário mínimo. O resultado do cenário 1 indica reduzida MB, descapitalização da atividade (ML negativa) e não remuneração dos custos de oportunidade (L negativo).

O Cenário 2 (Otimista) foi gerado pelos preços médios de compra dos animais, subtraídos do percentual do coeficiente de variação $(-12 \%)^{8}$, adicionando o percentual do coeficiente de variação ao preço de venda $(+5 \%)^{5}$ e subtraindo o percentual do coeficiente de variação ao salário mínimo médio $(-5,68 \%)^{9}$.

A interpretação deste cenário, em termos dos indicadores econômicos, é a mesma dos resultados determinístico (Tabela 1), porém em maior magnitude.

O Cenário 3 (Pessimista) foi obtido dos preços médios de compra dos animais, acrescidos do percentual do coeficiente de variação $(+12 \%)$, subtraindo o percentual do coeficiente de variação do preço de venda ($5 \%$ ) e adicionando o percentual do coeficiente de variação ao salário mínimo médio $(+5,68 \%)$. Interpreta-se a MB negativa como a incapacidade de pagamento das despesas operacionais, sendo a atividade completamente inviável.

O Cenário 4 (Melhores) foi obtido pelo preço mínimo de compra dos animais, pelo preço de venda máximo e pelo mínimo valor do salário mínimo. Nota-se que este cenário, ao retratar uma combinação de melhores valores para as três variáveis em questão, faz com que a atividade seja lucrativa e atrativa.

Coeficiente de variação calculado a partir da série de preços coletados.

9 Coeficiente de variação calculado a partir da série de salários mínimos coletados. 


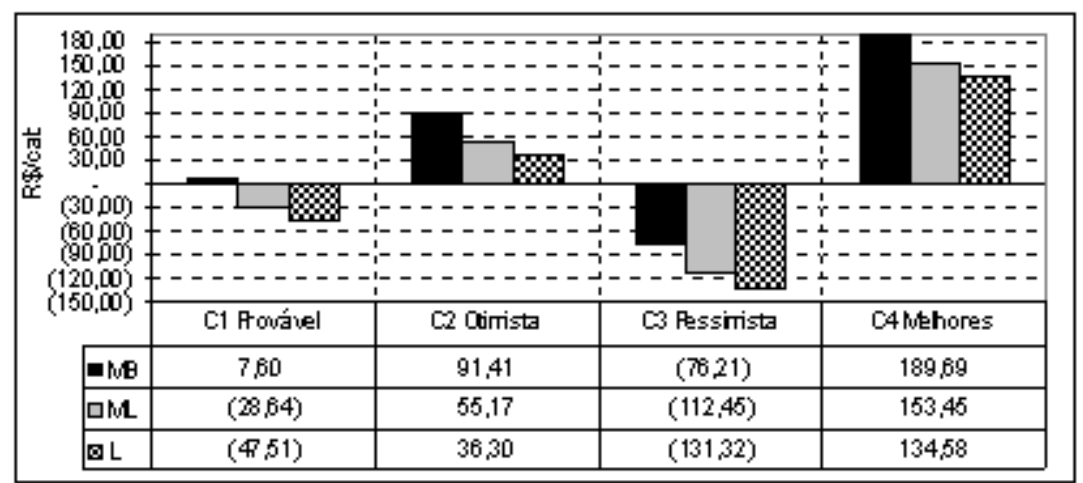

Gráfico 1 - Diferentes cenários encontrados para as variáveis de saída, Margem Bruta (MB), Margem Líquida (ML) e Lucro (L).

Fonte: Dados da pesquisa.

Por meio da observação conjunta dos vários cenários elaborados, pode-se perceber que, em termos médios (Cenário Médio), a atividade desenvolvida dentro dos padrões e tecnologia encontrados no centro de pesquisa de Aquidauana não proporciona condição de sustentabilidade do negócio no longo prazo e cobre, com pouca margem, os custos variáveis de produção. Isto significa que, para preços médios pagos pelos bezerros e para preços médios recebidos pelas vendas, há necessidade de maior eficiência no uso dos fatores de produção, para que essa situação de ML e L negativo seja evitada. A comparação com os demais cenários mostra, claramente, essa situação próxima do "ponto de fechamento" $(\mathrm{MB}=0)$ da atividade pecuária, uma vez que, em relação ao cenário médio, se as condições de preço melhorassem (otimista), todos os indicadores se tornariam positivos, enquanto se a situação revertesse para uma condição desfavorável (pessimista), ter-se-ia a indicação de que o abandono desta atividade seria a melhor decisão econômica a ser tomada.

Este método de avaliação comparativa de diferentes cenários permite fazer algumas inferências sobre possíveis situações futuras; entretanto, não são consideradas todas as possibilidades de ocorrência dos valores 
para as variáveis de entrada, bem como não se pode prever a probabilidade de ocorrência de cada um deles. Em outras palavras, a análise mostra o que pode acontecer, mas não "garante" que um dos cenários possa ocorrer. Mesmo que se aumente o número de variáveis simuladas, com o intuito de obter maior acurácia dos cenários, a análise permanecerá estática e com poder limitado de fornecimento de informações para uma tomada de decisão mais consciente.

\subsection{Análise de risco}

Finalmente, a análise de risco vem acrescentar informações relevantes à tomada de decisão e superar algumas limitações da análise estática de cenários e da análise determinística de resultados. Na simulação de risco foram utilizadas as mesmas variáveis de entrada da análise de cenários, ou seja, preço de compra, de venda dos animais e salário mínimo pago.

Os resultados desta análise resumem-se nos três gráficos apresentados a seguir. O Gráfico 2 diz respeito à Margem Bruta; o Gráfico 3, à Margem Líquida; e o Gráfico 4, ao Lucro.

Após obtenção dos resultados dos indicadores econômicos, foram encontradas as distribuições de frequiência das séries históricas dos preços pagos pelos bezerros, dos preços recebidos pelos animais vendidos no final do período e do salário mínimo. A partir destas distribuições, foram geradas 2000 simulações, das quais originaram as frequiências acumuladas de probabilidade para as três variáveis de análise (MB, ML e L), plotadas nos referidos gráficos.

Assim, o Gráfico 2 mostra que há probabilidade de a MB do sistema de produção de recria de gado de corte estudado ser negativa $(<0)$, da ordem de $47,6 \%$. A título de exemplo, existe a probabilidade de, aproximadamente, $50 \%$ da MB ser menor que $\mathrm{R} \$ 10,00 / \mathrm{cab}$. 


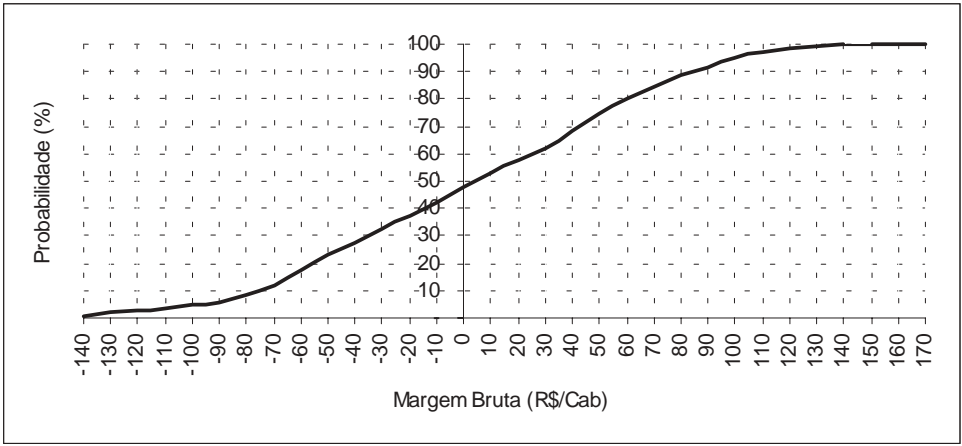

Gráfico 2 - Probabilidade acumulada de ocorrência dos valores de Margem Bruta no sistema de recria de gado de corte em pastejo rotacionado na região de Aquidauana - MS, 2005.

Fonte: Dados da pesquisa.

O Gráfico 3 indica que a ML tem $63,1 \%$ de probabilidade de se tornar negativa, o que indica grande risco de o sistema de produção se descapitalizar ao longo de sua vida útil. Considerando os valores encontrados, seria arriscado manter a atividade, sob pena de não obter sustentabilidade no longo prazo.

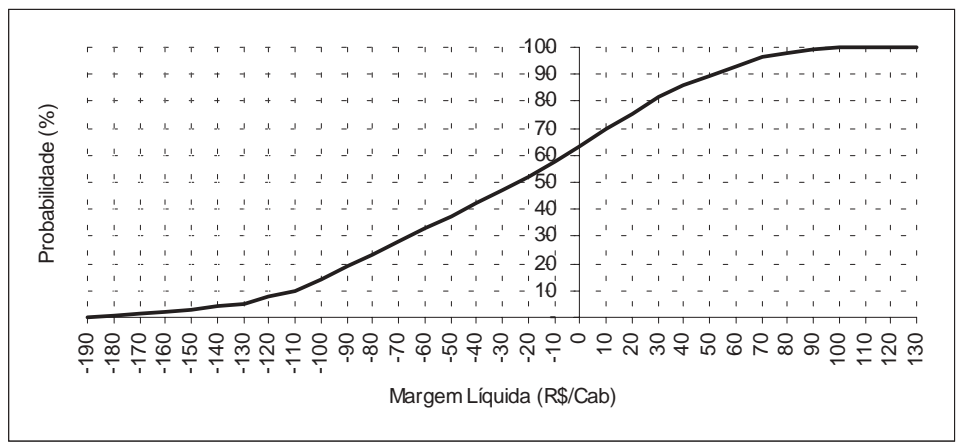

Gráfico 3 - Probabilidade acumulada de ocorrência dos valores de Margem Líquida no sistema de recria de gado de corte em pastejo rotacionado na região de Aquidauana - MS, 2005.

Fonte: Dados da pesquisa. 
O Gráfico 4 mostra que há elevada probabilidade $(74,85 \%)$ de o sistema de produção não remunerar o capital médio empatado a uma taxa de juros de $6 \%$ ao ano, ou seja, não cobrir os custos de oportunidades de uso do recurso financeiro.

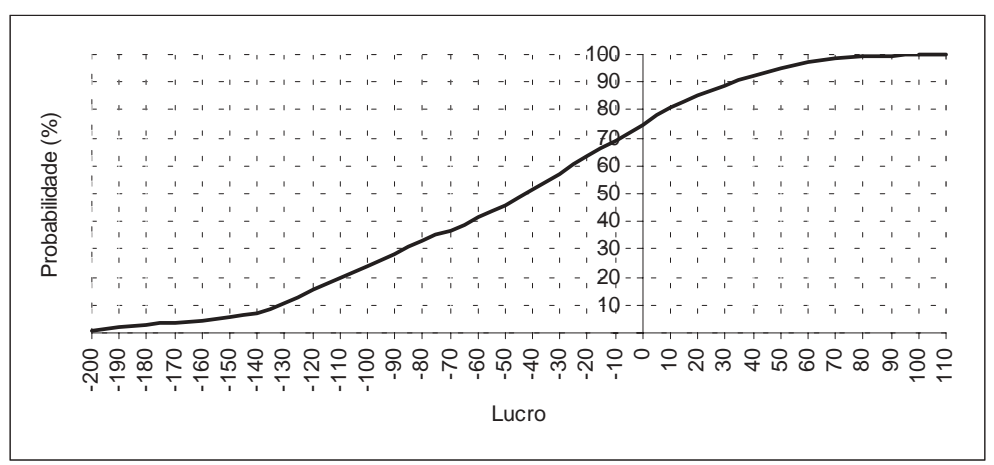

Gráfico 4 - Probabilidade acumulada de ocorrência dos valores de Lucro no sistema de recria de gado de corte em pastejo rotacionado na região de Aquidauana - MS, 2005.

Fonte: Dados da pesquisa.

Os resultados mostrados nos gráficos deixam clara a situação econômica desfavorável do sistema de produção de recria utilizado no estudo. Este sistema apresenta, aproximadamente, $47,6 \%$ de chance de não cobrir sequer os custos variáveis ( $\mathrm{MB}<0) ; 63,1 \%$ de não se manter sustentável no longo prazo, pelo pagamento dos custos operacionais e depreciação; e uma chance de apenas $25,15 \%$ (74,85\% subtraído de 100\% - Gráfico 4) de obtenção de lucro pelo menos igual a zero, a qual seria uma posição de equilíbrio, de acordo com a teoria econômica. 


\section{Conclusões}

Diante dos resultados apresentados, conclui-se que a primeira análise feita no estudo (cálculo dos resultados determinísticos - Tabela 1) aproximou-se mais do Cenário 2 (Otimista) do que do Cenário 1 (Cenário "Médio"). Por meio da simulação de Monte Carlo, pode-se observar que a situação retratada na Tabela 1 apresenta uma combinação entre preço relativamente baixo para compra dos animais e preço relativamente alto para venda. Tal fato revela que apenas a análise de resultado econômico, calculado de forma determinística, é insuficiente para captar variações relativas nas variáveis do modelo, as quais podem afetar o desempenho e a lucratividade da atividade. Não obstante, a análise simples de resultado econômico revela-se importante para o diagnóstico de eficiência de alocação de recursos e do uso adequado da tecnologia utilizada.

A geração de cenários mostra-se importante para a tomada de decisão, uma vez que possibilita a identificação de resultados diferenciados dos encontrados. Tal fato possibilita a comparação do resultado efetivo da atividade com outras possibilidades de ocorrência, sem que sejam necessárias comparações menos precisas com outros sistemas de produção. Este fato dá ao produtor rural uma noção de como está o andamento de seu negócio e também uma visão comparativa de como poderia ter sido o resultado, caso as variáveis de entrada analisadas não tivessem os mesmos valores analisados.

Mais importante do que conhecer os resultados de uma atividade e compará-la com diferentes possibilidades é poder reduzir os riscos e incertezas de um investimento ou até mesmo ajustar o sistema de produção para que ele incorra em menor probabilidade de resultados não desejáveis. A análise de risco possibilita identificar as probabilidades de ocorrências de resultados antieconômicos e, assim, nortear as decisões dentro de um nível razoável de segurança.

Com respeito aos números encontrados pelas análises, a principal conclusão que se pode ter é que a fase de recria da produção de gado de 


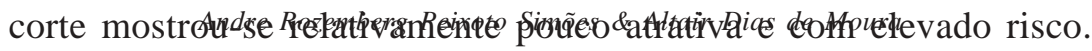
Dentre os fatos que levam a estes resultados, pode-se dizer que maiores escalas de produção gerariam ganhos de eficiência de utilização de recursos, podendo melhorar os indicadores encontrados. Da mesma forma, a existência de um planejamento adequado da produção, coordenado com uma estratégia de compra de insumos e venda do produto, poderia fazer com que o sistema operasse de maneira eficiente e na faixa de probabilidade de ocorrência de lucros positivos. Ressaltase, também, que o uso mais eficiente dos recursos aplicados na atividade, principalmente no que diz respeito à mão-de-obra contratada, associado ao aumento de escala a um nível tecnológico diferente, poderia proporcionar redução nos custos e, conseqüentemente, melhoria nos resultados.

Os resultados deste trabalho geram informações importantes para a tomada de decisão do pecuarista, visto que mostram que os processos de gerenciamento e de tomada de decisão podem ser bastante aprimorados, quando se colocam à disposição do tomador de decisão informações mais ricas e contextualizadas, mediante adoção de técnicas de simulação de cenários e riscos.

Como sugestão para trabalhos futuros, abre-se a possibilidade de se incluir maior número de variáveis de entrada nas simulações, pelo uso de análise de sensibilidade para sua identificação e busca de séries históricas que possam retratar seu comportamento. 


\section{Referências}

ALMEIDA E. F., TONELLI M. T. L., SANTOS O. B., SOUZA P. M., PONCIANO N. J., Análise Econômica para Implantação de um Projeto para Produção de Polpa de Frutas no Município de Campos dos Goytacazes - RJ. In: XLI Congresso Brasileiro Sociedade Brasileira de Sociologia e Economia Rural SOBER, 2003. Anais. Juiz de Fora, MG, 2003.

BONJOUR, S.C.M. Impactos estruturais de mudanças na preferência internacional de carne bovina. Viçosa: UFV, 2003. 114p. Tese (Doutorado em Economia Aplicada) - Universidade Federal de Viçosa, 2003.

BUARQUE, C. Avaliação econômica de projetos. 6 ed. Rio de Janeiro: Campus, 1991.266p.

Federação da Agricultura de Mato Grosso do Sul - FAMASUL. Banco de dados. www.famasul.gov.br Acessado em março 2005.

Instituto de Pesquisa Econômica Aplicada - IPEA. IPEADATA. www.ipeadata.gov.br acessado em março 2005.

MATSUnAGA, M. et alii. Metodologia de custo de produção utilizada pelo IEA. São Paulo, Agricultura em São Paulo 1976

MAYA F. L. A., Produtividade e Viabilidade Econômica da Recria e Engorda de Bovinos em Pastagens Adubadas Intensivamente Com e Sem o Uso da Irrigação. Piracicaba - 2003. 94 p. Dissertação (Mestrado em Agronomia). Escola Superior de Agricultura "Luiz de Queiroz", 2003.

PONCIANO N. J., SOUZA P. M., MATA H. T. C., VIEIRA J. R., Análise de Viabilidade Econômica e de Risco da Fruticultura na Região Norte Fluminense. In: XLI Congresso Brasileiro Sociedade Brasileira de Sociologia e Economia Rural SOBER, 2003. Anais. Juiz de Fora, MG, 2003 
REIS, J.D. Participação Brasileira no Mercado Internacional de Carne Bovina, 1990 - 2002. Viçosa: UFV, 2003. 100 p. Dissertação (Mestrado em Economia Aplicada) - Universidade Federal de Viçosa, 2003.

SANTOS H. N., VIEIRA W. C., ERU 528 Pesquisa Operacional Aplicada ao Agronegócio. MBA Gestão do Agronegócio. Universidade Federal de Viçosa. Viçosa: UFV. 2003.

SIMÕES A. R. P. Rastreabilidade da Carne Bovina como Condicionante da Rentabilidade da Cadeia Produtiva: Um Estudo de Caso no Estado de Goiás. Viçosa: UFV, 2003. 102 p. Dissertação (Mestrado em Economia Aplicada) - Universidade Federal de Viçosa, 2003.

SOUZA, J. L. M., Modelo para Análise de Risco Econômico Aplicado ao Planejamento de Projetos de Irrigação para a Cultura do Cafeeiro. Piracicaba - 2001. Dissertação (Doutorado em Agronomia). Escola Superior de Agricultura "Luiz de Queiroz”, 2001.

VARIAN, H.R. Microeconomia: princípios básicos. Rio de Janeiro: Campus, 2000.

Abstract - The success of the agricultural systems is affected by several social, cultural and economical variables, which can restrict or nurture the development of the agribusiness. In this context, agricultural managers search for ways to improve production efficiency. This effort comprises the improvement of the managerial activities and decision making process. This research aimed to build a risk analysis framework, using different scenarios of economical analysis of an animal husbandry firm. The data used was collected from a rotational grazing system for beef cattle. The results suggest that the profitability of the system is low, and some of the risk scenarios presented negative profitability.

Keywords: Economical analysis, beef cattle, scenarios, Monte Carlo simulation, risk analysis. 


\section{Apêndice}

Tabela 1a - Detalhamento de renda, custos e indicadores de resultado econômico encontrados para o sistema de produção de recria de machos em pastejo rotacionado na região de Aquidauana - MS, fevereiro 2005.

\begin{tabular}{|c|c|c|c|c|}
\hline \multirow[t]{2}{*}{ ESPECIFICAÇÃO } & \multirow{2}{*}{$\begin{array}{r}\text { Unidade Total da } \\
\text { Atividade } \\
\end{array}$} & \multicolumn{2}{|c|}{ Total por unidade } & \multirow[t]{2}{*}{$\% *$} \\
\hline & & $\mathbf{R} \$ / \mathbf{c a b}$ & R\$/ha & \\
\hline 1. RENDA BRUTA & $\mathrm{R} \$$ & & & \\
\hline Venda de animais & $22.928,00$ & 573,20 & $2.292,80$ & \\
\hline Outras receitas & $\mathrm{R} \$$ & & & \\
\hline TOTAL & $22.928,00$ & 573,20 & $2.292,80$ & $\begin{array}{l}100,00 \\
\%\end{array}$ \\
\hline
\end{tabular}

2. CUSTOS DE PRODUÇÃO:

2.1. CUSTO OPERACIONAL EFETIVO - COE:

\begin{tabular}{|c|c|c|c|c|c|c|}
\hline \multicolumn{2}{|c|}{ Compra de animais** } & $\mathrm{R} \$$ & $15.665,60$ & 391,64 & $1.566,56$ & $68,33 \%$ \\
\hline \multicolumn{2}{|l|}{ Sal mineral 1} & $\mathrm{R} \$$ & 589,60 & 14,74 & 58,96 & $2,57 \%$ \\
\hline \multicolumn{2}{|c|}{ Mão-de-obra contratada** } & $\mathrm{R} \$$ & 878,40 & 21,96 & 87,84 & $3,83 \%$ \\
\hline \multicolumn{2}{|c|}{ Assistência técnica*** } & $\mathrm{R} \$$ & $1.040,00$ & 26,00 & 104,00 & $4,54 \%$ \\
\hline \multicolumn{2}{|c|}{ Contabilidade** } & $\mathrm{R} \$$ & 260,00 & 6,50 & 26,00 & $1,13 \%$ \\
\hline \multicolumn{2}{|c|}{ Manutenção de pastagens } & $\mathrm{R} \$$ & 420,00 & 10,50 & 42,00 & $1,83 \%$ \\
\hline \multicolumn{2}{|c|}{ Vacinas e medicamentos } & $\mathrm{R} \$$ & 194,40 & 4,86 & 19,44 & $0,85 \%$ \\
\hline \multicolumn{2}{|l|}{ Energia } & $\mathrm{R} \$$ & 600,00 & 15,00 & 60,00 & $2,62 \%$ \\
\hline \multicolumn{2}{|l|}{ Combustível } & $\mathrm{R} \$$ & 800,00 & 20,00 & 80,00 & $3,49 \%$ \\
\hline \multicolumn{2}{|c|}{ TOTAL DO C.O.E } & $\mathbf{R} \$$ & $20.448,00$ & 511,20 & $2.044,80$ & $89,18 \%$ \\
\hline \multicolumn{7}{|c|}{ 2.2. CUSTO OPERACIONAL TOTAL - COT } \\
\hline \multicolumn{2}{|c|}{ Custo operacional efetivo } & $\mathrm{R} \$$ & $20.448,00$ & 511,20 & $2.044,80$ & $89,18 \%$ \\
\hline \multirow[t]{3}{*}{ Depreciação } & - benfeitorias & $\mathrm{R} \$$ & 955,41 & 23,89 & 95,54 & $4,17 \%$ \\
\hline & - animais de serviço & $\mathrm{R} \$$ & 142,86 & 3,57 & 14,29 & $0,62 \%$ \\
\hline & - forrageiras não anuais & $\mathrm{R} \$$ & 351,27 & 8,78 & 35,13 & $1,53 \%$ \\
\hline \multicolumn{2}{|l|}{ TOTAL C.O.T } & $\mathbf{R} \$$ & $21.897,54$ & 547,44 & $2.189,75$ & $95,51 \%$ \\
\hline
\end{tabular}


Andre Rozemberg Peixoto Simões \& Altair Dias de Moura

\subsection{CUSTO TOTAL}

Custo operacional total

R\$ $\quad 21.897,54 \quad 547,44 \quad 2.189,75 \quad 95,51 \%$

Remuneração do capital - benfeitorias

$\begin{array}{llll}\mathrm{R} \$ & 596,73 \quad 14,92 \quad 59,67 & 2,60 \%\end{array}$

- forrageiras não anuais

$\begin{array}{lllll}\mathrm{R} \$ & 158,07 & 3,95 & 15,81 & 0,69 \%\end{array}$

CUSTO TOTAL - C.T

R\$ $\quad 22.652,34 \quad 566,31 \quad 2.265,23 \quad 98,80 \%$

3. INDICADORES DE RESULTADOS:

\begin{tabular}{|c|c|c|c|c|}
\hline 3.1. Margem bruta total (RB-COE) & $\mathrm{R} \$$ & $2.480,00$ & 62,00 & 248,00 \\
\hline 3.2. Margem líquida total (RB-COT) & $\mathrm{R} \$$ & $1.030,46$ & 25,76 & 103,05 \\
\hline 3.3. Lucro total (RB-CT) & $\mathrm{R} \$$ & 275,66 & 6,89 & 27,57 \\
\hline
\end{tabular}

* Percentagem dos custos sobre a receita total

** Variáveis utilizadas nas simulações de cenários e de risco 
REVISTA DE ECONOMIA E AGRONEGÓCIO, VOL.4, $N^{o} 1$ 